\title{
Study of a Hybrid OCDMA-WDM Segmented Ring for Metropolitan Area Networks
}

\author{
G. Rodríguez de los Santos, J. A. Hernández, M. Urueña, I. Seoane, D. Larrabeiti \\ Dept. Ing. Telemática, Universidad Carlos III de Madrid \\ Avda Universidad 30, E-28914 Leganés, Madrid, Spain \\ Email: \{gsantos, jahgutie, muruenya, iseoane, dlarra\}@it.uc3m.es
}

\begin{abstract}
Optical Code Division Multiple Access (OCDMA) techniques have shown outstanding capabilities in the sharing of optical media, in particular in access networks. However, OCDMA systems may suffer from Multiple Access Interference (MAI) and other kinds of noise when many users access the shared media simultaneously, increasing the BER (Binary Error Rate) to unacceptable levels, that is, a situation at which all combined signals interfere and are lost.

This work proposes a mixed OCDMA and Tunable TransmitterFixed Receiver (TT-FR) WDM and ring architecture at which the ring is split into small-size segments to limit the probability of MAI. Essentially, every segment in the ring has got two hub nodes (on the segment's head and tail) which forwards inter-segment traffic to other hub nodes on dedicated home wavelengths, thus making use of WDM. The access media inside the segment is shared between the nodes by means of OCDMA, and code reuse is possible on different segments. Our performance analysis shows how to split a given ring into segments in order to minimise the BER due to multiple users accessing the network and allow for high bit-rates for a given traffic load. In addition, we analyse the possibility of introducing Forward Error Correction (FEC) at a moderate overhead cost to improve performance.
\end{abstract}

\section{INTRODUCTION AND RELATED WORK}

Code Division Multiple Access (CDMA) [1], [2] techniques have proven to offer higher capacity than Wavelength-Routed Networks (WRN) thanks to the statistical multiplexing properties they offer [3]. Indeed, the use of orthogonal codes allows multiple users to simultaneously transmit on the same frequency band without interfering with each other [1], [2]. However, OCDMA suffers from Multiple Access Interference (MAI), which often arises when more than a given number of users access the shared media simultaneously. This may happen both when we have pseudo-orthogonal (incoherent OCDMA) and theoretically orthogonal (either coherent or incoherent with differential detection) codes. When this occurs, the signal quality drops at the receivers, and all colliding symbols cannot be successfully decoded. This is often referred to as an "outage", and retransmission of all the packets involved in the outage is required. Nevertheless, orthogonal and pseudo-orthogonal codes may achieve high-performance results as long as such MAI limit is not exceeded.

OCDMA techniques have been proposed for access networks (FTTx) [4], [5], metropolitan area networks [6], [7], [8], [9], [10], [11] and backbone networks [12], [13], [14], [15]. For instance, OCDMA codes are very suitable for simplifying the Medium Access Control of the upstream channel in PassiveOptical Networks (PONs). At present, the OLT (Optical Line Terminal) coordinates the access to the upstream channel by the ONUs (Optical Network Units), granting access to them at specific non-overlapping timeslots. OCDMA techniques would allow the users to simultaneously access the channel without interfering each other as long as an acceptable MAI probability limit is not exceeded. In the case of backbone networks, orthogonal codes have been proposed to be used as labels in GMPLS networks [12], [13] on attempts to increase the capacity provided by optical fibres due to statistical multiplexing.

Finally, concerning optical ring architectures for Metropolitan Area Networks (MAN), most of today's proposals are based on transparent optical WDM ring networks at which each node in the ring is often offered a dedicated home wavelength for reception, see for instance Hornet, Mawson, RingO and DBORN [16], [17], [18]. Additionally, transmission collisions (when two or more users aim to transmit on the same wavelength of a third node) are avoided by defining Medium Access Control (MAC) protocols that arbitrate channel access, either by using a dedicated control channel, by delaying and inspecting wavelength use [18] or by circulating a token around the ring [19].

Although such TT-FR architectures are transparent and simple to deploy, substantial bandwidth capacity is wasted if nodes have sub-wavelength demands or traffic matrices are very asymmetric, that is, if some nodes have most of the time their reception wavelengths idle, since other nodes do not use them. OCDMA permits bandwidth reuse since all the resources (bandwidth capacity) are shared by all users. There have been some proposals regarding OCDMA-based ring configurations but these are quite limited by MAI because of packet recirculation through the ring [20], leading to self interference. A possible solution to this issue considers to implement code add/drop multiplexers, but this strategy requires either network synchronisation [21], additional parallel optical sources [22] or taking into account special properties of optical codes [7] which might not be possible for many code families. For this reason, we propose a hybrid OCDMA-WDM segmented ring architecture at which the inter-segment communication follows the same principles of TT-FR WDM rings, while the intrasegment communication occurs in the OCDMA domain. This 
architecture and its performance evaluation in terms of MAI probability is presented next.

\section{THE HYBRID WDM-OCDMA RING ARCHITECTURE}

Let us consider the $N=16$ node unidirectional (clockwise) OCDMA ring of Fig. 1(a). This ring is split into a number $K$ of segments ( $K=4$ for the ring of Fig. 1(a)) with $M$ nodes per segment. The nodes interfacing adjacent segments, referred to as hub nodes, are depicted with squares in the figure, in contrast to regular ones depicted with circles. Now, let us consider a single $M$-sized segment in the ring, see Fig. 2.

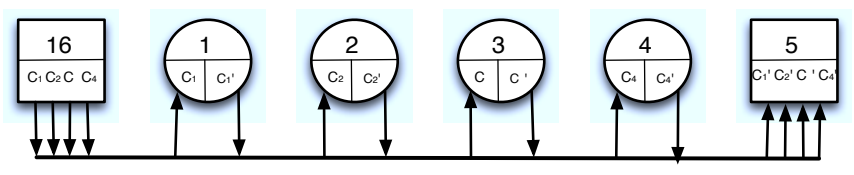

Fig. 2. OCDMA-WDM segment example $(M=4)$

We assume that each regular node $i$ in the ring has got a unique code for transmission and another one for reception, namely $\left(C_{i}^{\prime}, C_{i}\right)$ respectively. Following the testbed of [23], we assume that codes $C_{i}^{\prime}$ and $C_{i}$ use the same codeword but are encoded in orthogonal polarisations, thus not interfering with each other.

That is, every transit node in the segment has got one OCDMA encoder/decoder pair. The hub nodes have got $(M-1)$ encoders/decoder pairs interfacing respectively the next and previous segments they are attached to. The $(M-1)$ encoders of the hub node must match each of the decoders of the regular nodes, and viceversa. This way, the hub nodes have got a means to both collect the traffic from its inbound segment and send traffic to the outbound segment in the ring.

In addition to this, each hub node is provided with at least one home wavelength $\left(H W_{n}\right.$ in Fig. 1(a)) to receive packets from other hub nodes in the network, as well as one or more Tunable Transmitters that allow them to send packets to other segments in the ring. The number of wavelengths depends on the aggregated traffic generated by each segment. Thus, inter-segment traffic is exchanged between hub nodes in a TT-FR fashion, while intra-segment traffic is exchanged via OCDMA. Both the OCDMA and the WDMA parts have got a dedicated fibre on opposite circulation directions. In Fig. 1(a), for instance, the OCDMA circulation uses the clockwise direction while the WDMA direction is counter-clockwise. Finally, we do not assume any time multiplexion, thus each station is able to transmit asynchronously.

As an example of intra-segment packet delivery, consider a packet sent from node 3 to node 2 (Fig. 1(b)). To do so, node 3 must encode the packet using its transmission code $\left(C_{3}^{\prime}\right)$. Then, tail hub node 4 sends the packet to head hub node 16 through $H_{4}$. Finally, hub node 16 sends the packet encoded with code $C_{2}$, the reception code for node 2 . For inter-segment packet delivery, consider a packet sent from node 2 to node 14 (Fig. 1(c)). This packet is first encoded at node 2 with code $C_{2}^{\prime}$ and delivered at hub-node 4 . Then, hub-node 4 decodes the packet and transmits the packet all-optically to hub-node 12 by tuning its laser on the destination's segment home wavelength, that is, $H W_{3}$. Finally, hub node 12 encodes this packet with $C_{14}$ (which is the reception code of node 14) and forwards it locally on its segment towards node 14 .

The role of the hub nodes in this architecture is then two-fold:

- First, they strip packets off the ring. Otherwise packets would circulate indefinitely, causing self-interference and noise accumulation. Tail hub-nodes forward only undelivered packets or packets intended for other segments, thus removing already delivered traffic from the ring.

- Second, they partition the ring into small-size segments, limiting the maximum number of OCDMA users simultaneously accessing a given segment to $M$ nodes at most (instead of $N$ ). This has a clear impact on reducing the MAI, that might limit the intrasegment performance, as shown in the next section.

Finally, it is worth noticing that code reuse among segments is possible since an OCDMA packet encoded on a given segment is never visible on a different segment. Hence, the hybrid OCDMA-WDM architecture might be possible just with a code cardinality of $(M-1)$ different codewords ( $M$ is the segment size). That is, we need $(M-1)$ codewords in one polarisation and $(M-1)$ codewords in the other one. If there were not polarisation multiplexion, we would need a code cardinality of at least $2(M-1)$ codewords for $(M-1)$ nodes.

Concerning hardware requirements, this architecture requires one OCDMA encoder/decoder pair per regular node, and $(M-1)$ encoder/decoder pairs per hub node, that is, $2(M-1)$ encoder/decoder pairs per segment times $K=\frac{N}{M}$, the number of segments, that is:

$$
\frac{N}{M} 2(M-1) \approx 2 N
$$

encoder/decoder pairs for large $M$. That is, the hardware requirements depend mostly on the ring size, not on how nodes are arranged into segments (segment size). Thus, a number of $(M-1)$ different codewords may coexist on the segment for each polarisation, since packets may be encoded with codes $C_{1}, \ldots, C_{M-1}$ by the hub nodes in one polarisation or codes $C_{1}^{\prime}, \ldots, C_{M-1}^{\prime}$ by the regular nodes in the other polarisation.

Concerning the dimensioning of the WDM part of the ring, that is, the number of transceivers of the TT-FR ring, each hub node requires:

$$
K \cdot\left\lceil\frac{M \cdot R_{b, \mathrm{OCDMA}}}{R_{b, \mathrm{WDM}}}\right\rceil \approx N \frac{R_{b, \mathrm{OCDMA}}}{R_{b, \mathrm{WDM}}}
$$

where $R_{b, \text { OCDMA }}$ is the binary rate of the OCDMA segment (for instance, $10 \mathrm{Gbps}$ ) and $R_{b, \mathrm{WDM}}$ is the binary rate of a 


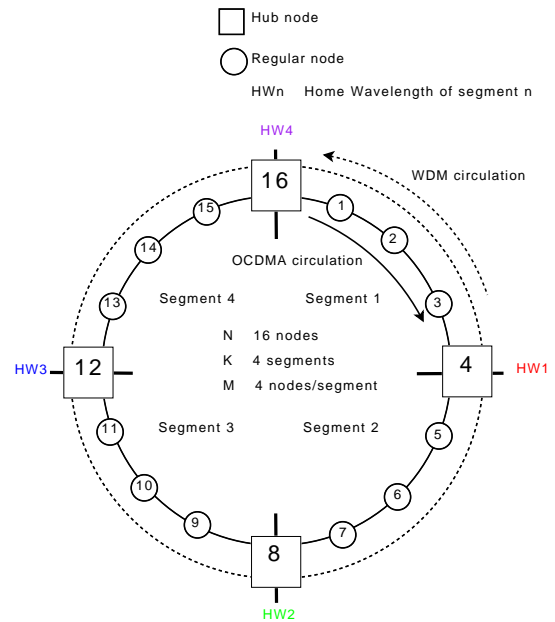

(a) OCDMA-WDM Ring example

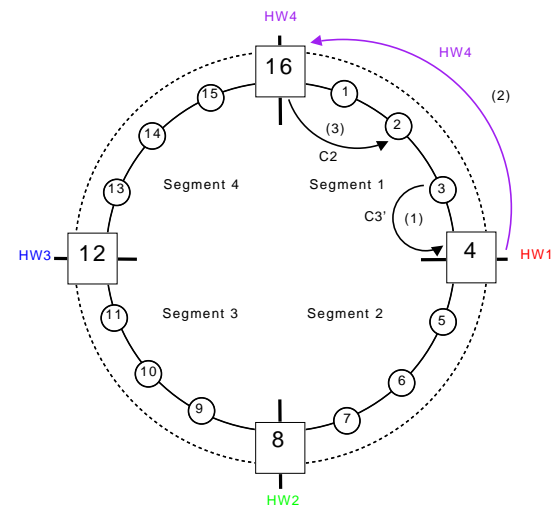

(b) Intra-segment delivery

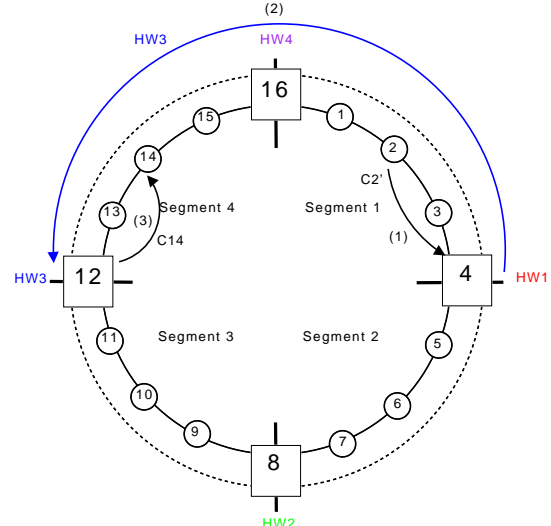

(c) Inter-segment delivery

Fig. 1. (a) Ring example, (b) Intra-segment delivery and (c) Inter-segment delivery of packets

WDM wavelength (for instance, $40 \mathrm{Gbps}$ ). Essentially, the hub nodes must collect the total traffic offered by the regular nodes (this is $M R_{b, \mathrm{OCDMA}}$ at most) and forward it to $K$ different segments.

Next, we analyse the probability to have MAI on a segment of the hybrid OCDMA-WDM architecture.

\section{ANALYSIS OF THE MAI PROBABILITY}

\section{A. MAI probability for $j$ active users}

This section derives the Bit Error Rate (BER) for a hybrid OCDMA-WDM ring with a total number of $N$ nodes and an arbitrary segment size $M$. Each coding technique has got its own BER equations for a number of $j$ active users. For performance evaluation of the OCDMA-WDM ring, we consider the Spectral Phase Encoding (a coherent OCDMA encoding) technique described in [24] (which was the inspiration for the testbed in [23]) because of its simplicity and feasibility. Nevertheless, it is worth noticing that this analysis may be performed for any other code family.

Now, let $A$ be a random variable that denotes the number of active codes in a given segment at a certain time (thus, with sample space $S_{A}=\{1, \ldots,(M-1)\}$. Additionally, let $I$ refer to the interference random variable which may take two possible values: $I=1$ if there is MAI, and $I=0$ otherwise. For the SPE system of [23] the MAI probability for $A=j$ active users is given by [24]:

$$
\begin{aligned}
P(I=1 \mid A=j) & =\frac{1}{2} \sum_{l=1}^{j-1}\left(\begin{array}{c}
j-1 \\
l
\end{array}\right)\left(\frac{1}{2 S}\right)^{l}\left(1-\frac{1}{2 S}\right)^{j-1-l} \\
& \times\left[1-\gamma^{\beta-1}(l)[\gamma(l)-\rho(l)]\right]
\end{aligned}
$$

where:

$$
\begin{aligned}
& \gamma(l)=1-e^{-\frac{I_{\text {thres }} N_{0}}{l P_{0}}} \\
& \rho(l)=1-Q\left(\sqrt{\frac{2 N_{0}}{l}}, \sqrt{\frac{2 N_{0} I_{\text {thres }}}{l P_{0}}}\right)
\end{aligned}
$$

Here, $Q(a, b)$ refers to the Marcum's $Q$ function:

$$
Q(a, b)=\int_{b}^{\infty} x \cdot \exp \left(\frac{-a^{2}-x^{2}}{2}\right) I_{0}(a x) d x
$$

where $I_{0}(x)$ is the modified Bessel function of the first kind and zeroth order.

We consider very similar parameters to those of the testbed in [23]. We assume $R_{b}=10 \mathrm{Gbps}$, a pulse width $\tau_{c}=450$ femtoseconds, a code cardinality of $N_{0}=64$. Here, $T_{b}$ is the bit period, then $S$ is computed such that $S=\frac{T_{b}}{T}$, where $T$ is the width of an encoded pulse, with $T=N_{0} \cdot \tau_{c}=28.8$ picoseconds. In our case, $S=\frac{T_{b}}{T}=3.47$. The bandwidth used is approximately $B W \approx 1 / \tau_{c}=2.22 \mathrm{THz}$. Additionally, $I_{\text {thres }}$ and $P_{0}$ are the threshold current for decision and the received power at the hub node respectively, since it is at the hub node where most interference may occur. Minimum BER is also achieved for ratios $I_{\text {thres }} / P_{0} \approx 0.4$, as noted in Fig. 9 of [24]. Finally, we consider that the receivers requires $\beta \cdot \tau_{c}$ seconds of time to output a bit decission.

\section{B. Probability of $A=j$ active users in an OCDMA segment}

Additionally, individual nodes are assumed to inject traffic to the segment following a Poisson process with an offered traffic of $a$ Erlang:

$$
a=\frac{\lambda}{\mu}
$$


where inter-arrival packet times are exponentially distributed with mean $1 / \lambda$, and service times are also exponentially distributed with mean $1 / \mu$. Following the Engset's analysis, the probability $b$ to have an active source, assuming its offered traffic is $a$, is given by:

$$
b=\frac{a}{1+a}
$$

Thus, in a given segment, the $(M-1)$ regular nodes inject $a$ Erlang of traffic. Next, we need to derive the amount of traffic injected by the $(M-1)$ encoders of the upstream hub node to each transit node in the segment. To do so, let $a_{i j}$ denote the amount of traffic offered by any node $i$ to any other node $j$ in the ring $(i, j=1, \ldots, N)$. Thus, assuming that all destination nodes are equally likely, then the total traffic destined to node $j$ in a given segment equals:

$$
\sum_{i, i \neq j} a_{i j}=\sum_{i, i \neq j} \frac{a}{N-1}=(N-1) \frac{a}{N-1}=a
$$

Hence, each code of the hub nodes inject $a$ Erlang of traffic, just like the codes of regular nodes. Thus, on a given segment, we have $2(M-1)$ equal sources of $a$ Erlang, $M-1$ of which are associated to the code space of the regular nodes and the other $M-1$ are associated to the code space of the hub nodes. It should be observed that only $M-1$ of those sources contribute to interference in each orthogonal polarisation, so only $M-1$ are relevant to our analysis. We analyse the probability of interference for any of the two polarisations. Thus, the probability to have exactly $j$ active sources out of $M-1$ possible on one polarisation is given by:

$$
P(A=j)=\left(\begin{array}{c}
M-1 \\
j
\end{array}\right) b^{j}(1-b)^{(M-1)-j}
$$

where $b$ follows eq. 7. Also, the average number of active interfering codes on a segment is $(M-1) b=(M-1) \frac{a}{1+a}$, which increases with the number of nodes per segment and traffic load $a$ Erlang per node. From a design point of view, the goal is to obtain the appropriate segment size $M$ to partition a ring such that the MAI probability on a segment is kept below some value, say for instance $10^{-9}$.

\section{Performance Analysis}

From an analysis perspective, we have considered a ring of an arbitrary size $N$ nodes but with different values of $M$ (nodes per segment) and $a$ Erlang of traffic, and studied the resulting segment BER, as:

$$
P(I=1)=\sum_{j=0} M-1 P(I=1 \mid A=j) P(A=j)
$$

following the total probability theorem.

In light of this, Fig. 3 shows the segment BER for $M=4,8$,
16 and 32 nodes for a ring with $N=64$ nodes. As expected, the segment BER increases with $M$ and $a$.

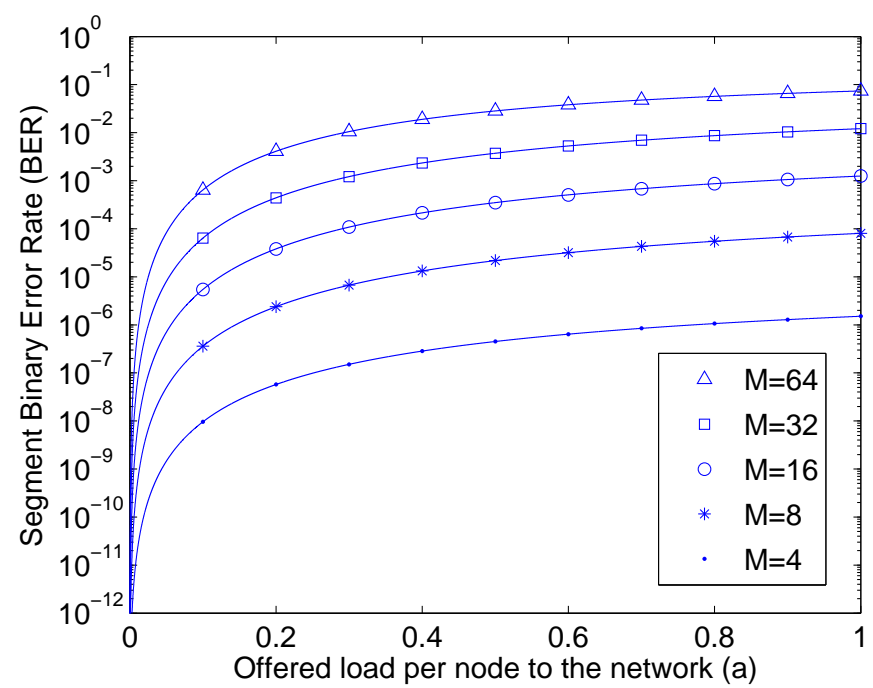

Fig. 3. Segment BER vs load for $N=64$ and $M=4,8,16,32$ for SPE

Fig. 3 also shows that doubling the number of nodes per segment implies one order of magnitude more in the BER probability at most traffic loads. That is, the segment BER at $a=0.2$ Erlang is about $10^{-6}$ for $M=8$ and drops to $10^{-5}$ for $M=16$, and $10^{-4}$ for $M=32$. In light of these results, the next design question is to study the maximum value of $M$ allowed for a segment size that satisfies a given MAI limit, say for example $P(I=1) \leq 10^{-9}$, for a given offered load of $a$ Erlang per node.

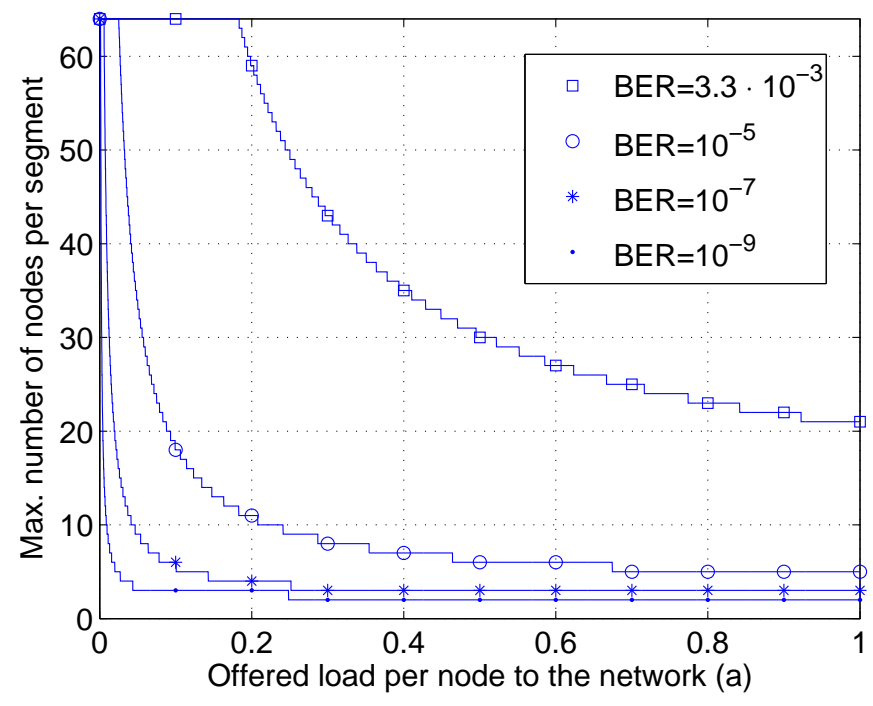

Fig. 4. Maximum segment size vs load for $N=64$ and $M=4,8,16,32$ for SPE method

In light of this, Fig. 4 shows the maximum segment size for different MAI limits $\left(3.3 \cdot 10^{-3}, 10^{-5}, 10^{-7}, 10^{-9}\right)$, at different traffic loads. For the BER limits of $10^{-9}$ and $10^{-7}$, the maximum segment size quickly decreases and a maximum 
of $M \leq 2$ or $M \leq 3$ respectively is allowed at network loads of roughly 0.25 Erlang (2.5 Gbps when $\left.R_{b, \text { OCDMA }}=10 \mathrm{Gbps}\right)$. A significant improvement is observed for a segment BER limit of $10^{-5}$ since, at the same load $a=0.25$ Erlang, $M \leq 10$ nodes is supported. The best results are shown for a BER limit of $3.3 \cdot 10^{-3}$ since $M \leq 64$ nodes ares supported at $a=0.183$ Erlang, $M \leq 50$ nodes for a $a=0.25$ Erlang and $M \leq 21$ nodes at full load: $a=1$ Erlang (that is, $10 \mathrm{Gbps}$ ). It should be noted that, even though the reference level for BER rate is $10^{-9}$, it is possible to reduce BER levels of $10^{-5}$ or even $3.3 \cdot 10^{-3}$ with Forward Error Correction (FEC) techniques at an overhead cost of $7 \%$ [25]. For instance, a BER limit of $3.3 \cdot 10^{-3}$ employing $7 \%$ FEC overhead is equivalent to a BER limit of $10^{-9}$ without any FEC.

Finally, concerning spectral efficiency, which we calculate as:

$$
\mathrm{Eff}=\frac{(M-1) \cdot a \cdot R_{b} \cdot\left(1-\eta_{F E C}\right)}{B W}
$$

where $\eta_{F E C}=0.07$ typically. Table I shows the spectral efficiency for a BER limit of $3.3 \cdot 10^{-3}$ at medium and full loads.

\begin{tabular}{|r|ccc|}
\hline BER Target & Load (Erlang) & \#Nodes & Eff $(\mathrm{bit} / \mathrm{s} / \mathrm{Hz})$ \\
\hline $3.3 \cdot 10^{-3}$ & 0.3 & 43 & 0.1055 \\
$3.3 \cdot 10^{-3}$ & 1 & 21 & 0.167 \\
\hline
\end{tabular}

TABLE I

SPECTRAL EFFICIENCY

\section{CONCLUSIONS}

This work has proposed a novel OCDMA-WDM ring architecture where the ring is split into a number of segments whose bandwidth is shared locally using OCDMA, while intersegment communication is performed using WDMA. Such a partitioning of the ring into segments permits:

- To limit the maximum number of codes under simultaneous transmission, thus reducing the probability of Multiple Access Interference.

- To reuse code words in other segments, thus reducing the OCDMA code cardinality significantly.

In terms of hardware, the number of encoder/decoder pairs in the ring regular node must be equipped just with one OCDMA code encoder/decoder pair. Additionally, the hub nodes also require $M-1$ OCDMA encoders and $M-1$ OCDMA decoders that is, as many encoders and decoders as nodes per segment for local traffic delivery; plus one or more WDM Tunable Transmitters and Fixed Receivers for inter-segment communication. Additionally, the hub nodes are required to decode and forward all the packets destined to other segments in the ring, which might be a processing burden. The performance analysis shows how to choose the maximum number of nodes per segment $M$ for a target BER probability under different traffic conditions. We have performed the analysis for the Spectral Phase Encoding technique of [24] with the parameters used in the testbed [23]). The simulation experiments have shown that employing FEC techniques with about $7 \%$ overhead may allow segments of up to 50 nodes at medium traffic $(25 \%)$ loads and 21 nodes at full load (100\%).

\section{ACKNOWLEDGEMENTS}

The work described in this paper was carried out with the support of the BONE project ("Building the Future Optical Network in Europe"), a Network of Excellence funded by the European Commission through the 7th ICT-Framework Programme.

Additionally, the authors would like to thank the support of the T2C2 Spanish project (under code TIN2008-06739-C04-01) and the Greencom UC3M-CAM project under code (CCG10UC3M/TIC-5624).

\section{REFERENCES}

[1] K. Fouli and M. Maier, "OCDMA and Optical Coding: Principles, Applications and Challenges," IEEE Communications Magazine, vol. 45, no. 8, pp. 27-43, Aug. 2007.

[2] H. Yin, D.J. Richardson, Optical Code Division Multiple Access Communication Networks. Theory and Applications. Beijing/New York: Springer, 2007.

[3] S. Goldberg and P. R. Prucnal, "On the teletraffic capacity of Optical CDMA," IEEE Trans. Communications, vol. 55, no. 7, pp. 1334-1343, Jul. 2007.

[4] J. Kani, K. Iwatsuki and T. Imai, "Optical Multiplexing Technologies for Access-Area Applications," IEEE Journal on Selected Topics in Quantum Electronics, vol. 12, no. 4, pp. 661-668, Jul.-Aug. 2006.

[5] K. Kitayama, X. Wang and N. Wada, "OCDMA Over WDM PONSolution Path to Gigabit-Symmetric FTTH," IEEE Journal of Lightwave Technology, vol. 24, no. 4, pp. 1654-1662, Apr. 2006.

[6] C.S. Bres, I. Glesk, R.J. Runser and P.R. Prucnal, "All Optical OCDMA Code Drop Unit for Transparent Ring Networks," Lasers and ElectroOptics Society, 2004. LEOS 2004. The 17th Annual Meeting of the IEEE, vol. 2, pp. 501-502, 7-11 Nov. 2004.

[7] C.S. Bres, I. Glesk, R.J. Runser and P.R. Prucnal, "All-Optical OCDMA Code-Drop Unit for Transparent Ring Networks," IEEE Photonics Technology Letters, vol. 17, no. 5, pp. 1088-1090, May. 2005.

[8] Y. Deng, Z. Wang, K. Kravtsov, J. Chang, C. Hartzell, M.P. Fok and P.R. Prucnal, "Demonstration and Analysis of Asynchronous and Survivable Optical CDMA Ring Networks," IEEE/OSA Journal of Optical Communications and Networking, vol. 2, no. 4, pp. 159-165, Apr. 2010.

[9] K. Kravtsov, Y. Deng, and P.R. Prucnal, "Self-Clocked All-Optical Add/Drop Multiplexer for Asynchronous CDMA Ring Networks," IEEE Journal of Quantum Electronics, vol. 45, no. 4, pp. 396-401, Apr. 2009.

[10] M. Gharaei, S. Cordette, I. Fsaifes, C. Lepers and P. Gallion, "A Novel Ring Architecture of Multiple Optical Private Networks over EPON using OCDMA Technique," 11th International Conference on Transparent Optical Networks, 2009. ICTON '09., pp. 1-4, Jun. 28 2009-Jul. 22009.

[11] B. Chen, C. Guo, J. Chen, L. Zhang, Q. Jang and S. He, "Add/drop Multiplexing and TDM Signal Transmission in an Optical CDMA Ring Network," OSA Journal of Optical Networking., vol 6., no. 8, pp. 969974, Aug. 2007.

[12] T. Khattab and H. Alnuweiri, "Optical CDMA for All-Optical SubWavelength Switching in Core GMPLS Networks," IEEE Journal on Selected Areas in Communications., vol 25., no. 5, pp. 905-921, Jun. 2007. 
[13] T. Khattab and H. Alnuweiri, "Optical GMPLS Networks with Code Switch Capable Layer for Sub-Wavelength Switching," Global Telecommunications Conference, 2004. GLOBECOM '04. IEEE., vol 3., pp. 1786-1792, 29 Nov.-3 Dec. 2004.

[14] M. Meenakshi and I. Andonovic, "Code-Based All Optical Routing Using Two-Level Coding," IEEE Journal of Lightwave Technology., vol 24., no. 4, pp. 1627-1637, Apr. 2006.

[15] N. Calabretta, G. Contestabile, A. D'Errico and E. Ciaramella, "AllOptical Label Processing Techniques for Pure DPSK Optical Packets," IEEE Journal of Selected Topics in Quantum Electronics., vol 12., no. 4, pp. 686-696, Jul.-Aug. 2006.

[16] White, I.M., Rogge, M.S., Shrikhande, K., Kazovsky, L.G., "A Summary of the HORNET Project: A Next-Generation Metropolitan Area Network," IEEE Journal on Selected Areas in Communications, vol. 21, no. 9, pp. 1478-1494, November. 2003.

[17] Gaudino, R., "RINGO: Demonstration of a WDM Packet Network Architecture for Metro Applications," 2002. Proceedings of the 4th International Conference on Transparent Optical Networks (ICTON) 2002, vol. 1, pp. 77-80, August. 2002.

[18] Bouabdallah, N., Perros, H., "Cost-effective single-hub WDM ring networks: A proposal and Analysis," Computer Networks, vol. 51, issue 13, pp. 3878-3901, September. 2007.

[19] Maier, M., Optical Switching Networks. New York: Cambridge University Press, 2008.

[20] Y. Gao, Y. Wang, W. Ma, J. Wu and J. Lin, "Code-Division Multiple-
Access in an Optical Fiber LAN with Ring," International Conference on Communication Technology Proceedings, 2003. ICCT 2003., vol 1., pp. 743-747, 9-11 Apr. 2003.

[21] J. Wu and C. Lin, "Fiber-Optic Code Division Add-Drop Multiplexers," IEEE Journal of Lightwave Technology., vol 18., no. 6, pp. 819-824, Jun. 2000.

[22] R.E. Gordon and L.R. Chen, "Demonstration of All-Photonic Code Conversion in a Semiconductor Fiber Ring Laser for OCDMA Networks," Information Photonics, 2005. IP 2005. OSA Topical Meeting on, pp. 1-3, Jun. 2005.

[23] Hernandez, V.J., Cong, W., Hu, J., Yang, C., Fontaine, N.K., Scott, R.P., Ding, Z., Kolner, B.H., Heritage, J.P. and Yoo, S.J.B., "A 320-Gb/s Capacity (32-User x $10 \mathrm{~Gb} / \mathrm{s}$ ) SPECTS O-CDMA Network Testbed With Enhanced Spectral Efficiency Through Forward Error Correction," IEEE Journal of Lightwave Technology, vol. 25, issue 1, pp. 79-86. January 2007.

[24] Salehi, J.A., Weiner, A.M. and Heritage, J.P., "Coherent Ultrashort Light Pulse Code-Division Multiple Access Communication Systems," IEEE Journal of Lightwave Technology, vol. 8, issue 3, pp. 478-491. March 1990.

[25] Tychopoulos, A. and Koufopavlou, O. and Tomkos, I, "FEC in Optical Communications," IEEE Circuits and Devices Magazine, vol. 22, issue 6, pp. 79-86. November/December 2006. 